\title{
InGaAs heteroepitaxy on GaAs compliant substrates: X-ray diffraction evidence of enhanced relaxation and improved structural quality
}

\author{
P. D. Moran ${ }^{a)}$ and D. M. Hansen \\ Department of Chemical Engineering, University of Wisconsin, Madison, Wisconsin 53706 \\ R. J. Matyi \\ Department of Materials Science and Engineering, University of Wisconsin, Madison, Wisconsin 53706 \\ J. G. Cederberg \\ Department of Chemical Engineering, University of Wisconsin, Madison, Wisconsin 53706 \\ L. J. Mawst \\ Department of Electrical and Computer Engineering, University of Wisconsin, Madison, Wisconsin 53706 \\ T. F. Kuech \\ Department of Chemical Engineering, University of Wisconsin, Madison, Wisconsin 53706
}

(Received 21 January 1999; accepted for publication 14 July 1999)

\begin{abstract}
$\mathrm{In}_{0.44} \mathrm{Ga}_{0.56} \mathrm{As}$ (3\% mismatch) films $3 \mu \mathrm{m}$ thick were grown simultaneously on a conventional GaAs substrate, glass-bonded GaAs compliant substrates employing glasses of different viscosity, and a twist-bonded GaAs compliant substrate. High-resolution triple-crystal x-ray diffraction measurements of the breadth of the strain distribution in the films and atomic force microscopy measurements of the film's surface morphology were performed. The films grown on the glass-bonded compliant substrates exhibited a strain distribution whose breadth was narrowed by almost a factor of 2 and a surface roughness that decreased by a factor of 4 compared to the film simultaneously grown on the conventional substrate. These improvements in the film's structural quality were observed to be independent of the viscosity of the glass-bonding media over the range of viscosity investigated and were not observed to occur for the film grown on the twist-bonded substrate. (C) 1999 American Institute of Physics. [S0003-6951(99)01637-X]
\end{abstract}

Limits on the structural quality of an epitaxial film which is lattice mismatched to the substrate precludes many potential device applications of heteroepitaxial semiconductor structures. Thin mismatched films retain a pseudomorphic strain so as to match the lattice constant of the much thicker substrate in the plane of the interface. Relaxation of a mismatched film grown beyond some critical thickness, ${ }^{1} h_{c}$, which decreases with increasing mismatch, occurs at the expense of the film's structural quality. The film is eventually fully relaxed to its bulk lattice constant as it is grown beyond $h_{c}$ through the generation of an increasing number of misfit dislocations at the film/substrate interface and the subsequent propagation of threading dislocations into the film. ${ }^{2}$

The concept of a compliant substrate has been proposed as a method for modifying the relaxation behavior of the heterostructure. ${ }^{3}$ An ideal compliant substrate consists of a thin "template", layer mechanically decoupled from a thicker "handle" wafer. Calculations ${ }^{3-6}$ of the strain partitioning in such a system predict enhanced relaxation without structural degradation of a mismatched film grown to a thickness well beyond the conventional $h_{c}$ on a template whose thickness is less than or approximately equal to $h_{c}$.

Experimentally realized compliant substrates have not had template layers that are completely decoupled from the handle wafer. Two approaches to fabricating macroscopic compliant substrates have predominated. The first utilized a

a)Electronic mail: pmoran@itis.com metal $^{7}$ or glass ${ }^{8-11}$ media to join the template and handle. The second approach employed twist-bonded (TB) substrates, ${ }^{12,13}$ in which the template was directly bonded to the handle with an intentional azimuthal angular misorientation $\theta$. The exact influence of the critical template/handle interface on film relaxation is uncertain at present. In glassbonded substrates, the ability of the glass to flow under a shear strain has been proposed as a means to allow strain relaxation. ${ }^{9,14}$ This mechanism depends critically on the value of the glass viscosity, which can be altered by temperature and composition. ${ }^{9}$ The angular misorientation present in a TB substrate, ideally determining an interface of screw dislocations, has been proposed to control the strain relaxation in such structures. ${ }^{12,15}$ It has been proposed that $\theta$ should be chosen to be a low symmetry angle so as to minimize the density of coincidence $\operatorname{sites}^{16}$ and that ${ }^{15} 2 \sin (\theta / 2)$ $>\epsilon$, where $\epsilon$ is the mismatch between the film and substrate.

Several studies utilizing transmission electron microscopy (TEM) have demonstrated a reduced dislocation density over sub-micron-sized areas of films when grown on glass-bonded ${ }^{8-10}$ and twist-bonded ${ }^{12,13}$ compliant substrates. There are comparatively little high-resolution x-ray diffraction (HRXD) data which measures the film relaxation behavior over macroscopic dimensions. One report ${ }^{17}$ of an $\mathrm{InSb}$ film grown with a very low dislocation density as observed by TEM on a TB GaAs substrate referred to a strain distribution and mosaic spread of the film measured by HRXD but did not compare these values with HRXD data of the same film grown on the conventional substrate. Previously pub- 
lished HRXD data from films grown beyond their critical thickness on the other types of compliant substrates have been interpreted in some studies as showing that the films retained more strain $^{7,18}$ and, in other annealing studies, less strain, 8,9 than films grown directly on conventional substrates. Increases in the breadth of the films' diffraction peak were observed to accompany relaxation on compliant substrates in these studies, indicating a potential degradation in the film's structural quality that further complicates interpretation of the data.

It is difficult to draw general conclusions as to the factors that determine the effect of substrate compliance through comparison of these previously reported results. Films of different degrees of mismatch were grown on compliant substrates that differ both in the thickness of the template layer and the type of bonding media employed. HRXD data measuring the effect of substrate compliance on the macroscopic scale are sparse and indicate that relaxation on a compliant substrate, if it occurs, may result in structural degradation of the film.

The present work compares the macroscopic relaxation behavior of $\operatorname{In}_{x} \mathrm{Ga}_{1-x} \mathrm{As}$ films grown simultaneously on GaAs-based compliant substrates and on conventional substrates. The template layer thickness and growth conditions are kept constant while the type of bonding media employed in the compliant substrate is varied. The heterostructure studied is one in which the film mismatch and thickness result in a highly relaxed film when grown on both a conventional and compliant substrate. Differences between the films are investigated by comparing peak breadths in two-dimensional HRXD data and surface morphology by atomic force microscopy (AFM).

The compliant substrates consisted of a $10 \mathrm{~nm} \mathrm{GaAs}$ growth template bonded to a GaAs handle wafer fabricated through a process reported elsewhere. ${ }^{11}$ Three compliant substrates were studied: a twist-bonded substrate with $\theta$ $\sim 12^{\circ}$, and two glass-bonded substrates employing either 10 $\mathrm{mol} \% \mathrm{~B}_{2} \mathrm{O}_{3}$ or $30 \mathrm{~mol} \% \mathrm{~B}_{2} \mathrm{O}_{3}$ borosilicate glass. An extrapolation of existing viscosity data for borosilicate glasses ${ }^{19}$ gives rise to an estimate that the viscosity of the 10 mol \% glass is $\sim 10^{17} \mathrm{P}$ at $650{ }^{\circ} \mathrm{C}$ and that of the $30 \mathrm{~mol} \%$ glass to be $\sim 10^{12} \mathrm{P}$. The results of simultaneous growth on these compliant substrates can, therefore, contrast the effects induced by a $\theta=12^{\circ}$ twist-bonded interface, the presence of a glass-bonded interface, and any effects due to a difference in glass viscosity of $\sim 5$ orders of magnitude.

$\mathrm{In}_{0.44} \mathrm{Ga}_{0.56} \mathrm{As}$ (3.0\% mismatch) films were simultaneously grown on a conventional substrate and these compliant substrates in a horizontal metal-organic vapor-phase epitaxy (MOVPE) reactor at 78 Torr and $650{ }^{\circ} \mathrm{C}$. The films were grown at $50 \mathrm{~nm} / \mathrm{min}$ to a thickness of $3 \pm 0.3 \mu \mathrm{m}$. X-ray diffraction analyses of the films employed a Bede $\mathrm{D}^{3}$ diffractometer and $\mathrm{Cu} K \alpha$ radiation. The beam was conditioned by a four-bounce $\mathrm{Si}$ (220) monochromator before impinging on the sample and defined by a four-bounce $\mathrm{Si}$ (111)-grooved crystal after diffracting from the sample. The area of the sample probed by the X-ray beam was approximately $1 \mathrm{~cm}$ by $1 \mathrm{~mm}$. The use of this triple-crystal diffractometer (TCD) allows the region of reciprocal space in the vicinity of the 004 points of the film and substrate to be mapped in two Downloaded 09 Jan 2007 to 128.104.198.71. Redistribution subje

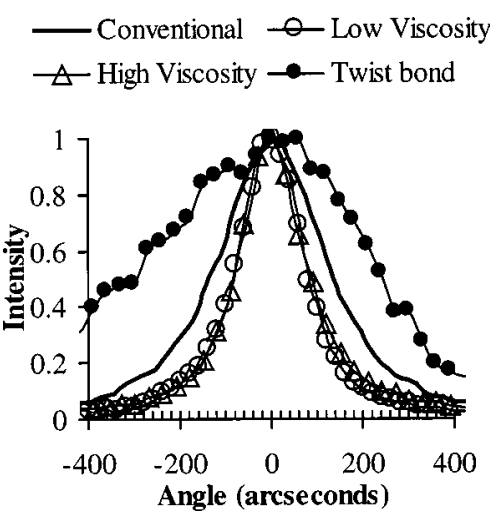

FIG. 1. Diffraction spectra along $q_{\|}$from the $\operatorname{In}_{0.44} \mathrm{Ga}_{0.56} \mathrm{As}$ (3\% mismatch) films demonstrate that the breadth of the strain distribution in films grown on glass-bonded compliant substrates is significantly narrower than in the film grown on the conventional substrate.

dimensions of reciprocal space. The direction parallel to the 004 diffraction vector is denoted by $q_{\|}$, and that perpendicular to the 004 diffraction vector is $q_{\perp}$. The intensity distribution along $q_{\|}\left(q_{\perp}\right)$ measures the lattice constant in the [001] direction (angular misorientation in the [110] direction) so that the peak breadth along $q_{\|}\left(q_{\perp}\right)$ is a measure of the strain distribution (mosaic spread) present in the film. The strain distribution in the film is measured independent of the film's misorientation and mosaic spread by investigating the distribution of intensity along $q_{\|}$after it has been integrated over the $q_{\perp}$ direction of reciprocal space. The large vertical divergence of a TCD in the third dimension of reciprocal space $\left(\sim 0.5^{\circ}\right)$ can effect the shape of profiles in the $q_{\perp}$ direction of reciprocal space for samples with a mosaic spread larger than $0.5^{\circ},{ }^{20}$ but has a negligible effect on peak widths recorded along $q_{\|}$for samples whose mosaic spread is less than $0.5^{\circ}$ due to the independence of the out-of-plane resolution function and scattering angle in the plane of the diffractometer. ${ }^{21}$

Figure 1 shows the distribution of diffracted intensity along $q_{\|}$from the $3 \%$ mismatched $\operatorname{In}_{0.44} \mathrm{Ga}_{0.56} \mathrm{As}$ films. Growth on high- and low-viscosity glass-bonded compliant substrates led to a film with a strain distribution resulting in a diffraction peak having a full width at half maximum $(F W H M)=160$ arcsec, significantly narrower than the $\mathrm{FWHM}=280$ arcsec of that due to the strain distribution seen in the growth on the conventional substrate. Growth on the $12^{\circ}$ TB compliant substrate resulted in a film strain distribution with a FWHM $=540$ arcsec significantly broader than that observed in the growth on the conventional substrate.

The use of a TCD to measure the strain distribution along $q_{\|}$resulted in the simultaneous collection of data on the films' degree of misorientation in the [110] direction lying in the plane of the diffractometer $\left(q_{\perp}\right)$. Observation of a tilt between the mechanical handle wafer and films grown on compliant substrates $\left(<0.1^{\circ}\right)$ was consistent with the expected difference in the surface normal of the two nominally (001) wafers bonded to fabricate the substrate. The tilt's impact on the measured strain distribution along $q_{\|}$after integration along $q_{\perp}$ is negligible. No attempt was made to probe the anisotropy in mosaic distribution in a single sample along two perpendicular [110] directions in the plane of the interface. However, differences between the mosaic spread of to AlP license or copyright, see http://apl.aip.org/apl/copyright.jsp 


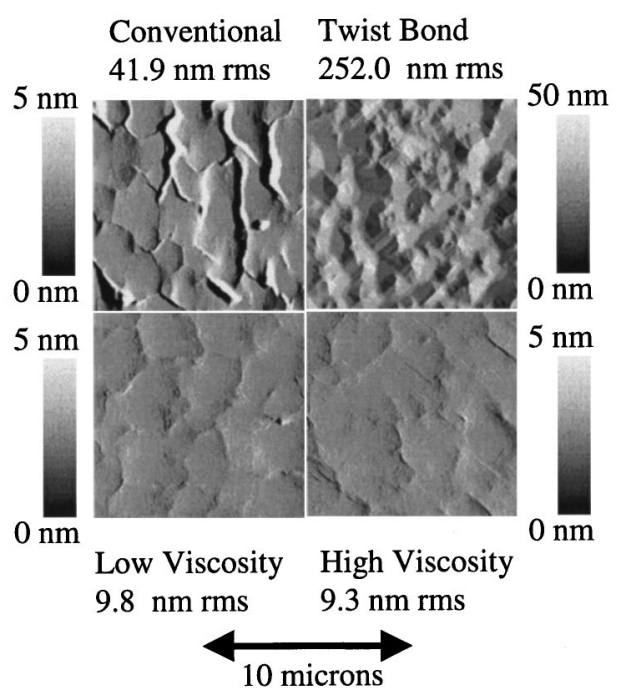

FIG. 2. AFM tip-deflection images of the $\operatorname{In}_{0.44} \mathrm{Ga}_{0.56} \mathrm{As}$ (3\% mismatch) films grown on conventional and compliant substrates indicate that growth on the glass-bonded substrates results in a significant reduction in the surface roughness.

samples along the one [110] direction $\left(q_{\perp}\right)$ in the plane of the diffractometer were not significant. The FWHM along $q_{\perp}$ for all films was within $20 \%$ of 1100 arcsec. The primary difference between films was in the breadth of their strain distribution.

The observed effects on the breadth of the strain distribution were coincident with atomic force microscopy observations of changes in the surface morphology of the films. Figure 2 shows tip-deflection images of the four $3 \%$ mismatched films. The rms surface roughness, measured by averaging three $10 \mu \mathrm{m} \times 10 \mu \mathrm{m}$ AFM micrographs, dramatically decreased for the films grown on glass-bonded compliant substrates, and dramatically increased for films grown on the $12^{\circ} \mathrm{TB}$ substrate. The failure of the TB substrate to improve the structural quality of the film may have been due to a degradation of the template layer during the substrate manufacturing process.

In summary, we have fabricated, grown on, and presented HRXD evidence of the compliance over macroscopic dimensions of glass-bonded GaAs compliant substrates. Two conclusions are consistent with these data. First, the growth on a glass-bonded compliant substrate of a highly mismatched film to well beyond its critical thickness results in a relaxed film with significantly narrower strain distribution and smoother surface than that achieved through growth on a conventional substrate. Second, the mechanism that is responsible for the observed enhancement of relaxation is insensitive to the viscosity of the bonding media in the range between $\sim 10^{17}$ and $\sim 10^{12} \mathrm{P}$.

This work is supported by the MURI program on compliant substrates under the Office of Naval Research (C. Wood). Facilities support by the NSF Materials Research Science and Engineering Center on Nanostructured Materials and Interfaces is gratefully acknowledged.

${ }^{1}$ J. W. Matthews, S. Mader, and T. B. Light, J. Appl. Phys. 41, 3800 (1970).

${ }^{2}$ B. W. Dodson and J. Y. Tsao, Appl. Phys. Lett. 51, 1325 (1987).

${ }^{3}$ Y. H. Lo, Appl. Phys. Lett. 59, 2311 (1991).

${ }^{4}$ D. Teng and Y. H. Lo, Appl. Phys. Lett. 62, 43 (1993).

${ }^{5}$ C. Carter-Coman, R. Bicknell-Tassius, A. Brown, and N. Jokerst, Appl. Phys. Lett. 71, 1344 (1997).

${ }^{6}$ L. B. Freund and W. D. Nix, Appl. Phys. Lett. 69, 173 (1996).

${ }^{7}$ C. Carter-Coman, R. Bicknell-Tassius, A. Brown, and N. Jokerst, Appl. Phys. Lett. 70, 1754 (1997).

${ }^{8}$ A. R. Powell, S. S. Iyer, and F. K. LeGoues, Appl. Phys. Lett. 64, 1856 (1994).

${ }^{9}$ M. O. Tanner, M. A. Chu, K. L. Wang, M. Meshkinpour, and M. S. Goorsky, J. Cryst. Growth 157, 121 (1995).

${ }^{10}$ Z. Yang, J. Alperin, W. I. Wang, S. S. Iyer, S. S. Kuan, and F. Semendy, J. Vac. Sci. Technol. B 16, 1489 (1998).

${ }^{11}$ D. M. Hansen, P. D. Moran, K. A. Dunn, S. E. Babcock, R. J. Matyi, and T. F. Kuech, J. Cryst. Growth 195, 144 (1998).

${ }^{12}$ F. E. Ejeckam, Y. H. Lo, S. Subramanian, H. Q. Hou, and B. E. Hammons, Appl. Phys. Lett. 70, 1685 (1997).

${ }^{13}$ F. E. Ejeckam, M. L. Seaford, Y. H. Lo, H. Q. Hou, and B. E. Hammons, Appl. Phys. Lett. 71, 776 (1997).

${ }^{14}$ M. A. Chu, M. O. Tanner, F. Y. Huang, K. L. Wang, G. G. Chu, and M. S. Goorsky, J. Cryst. Growth 175, 1278 (1997).

${ }^{15}$ T. Y. Tan and U. Gosele, Appl. Phys. A: Mater. Sci. Process. 64, 631 (1997).

${ }^{16}$ W. A. Jesser, J. H. van der Merwe, and P. M. Stoop, J. Appl. Phys. 85, 2129 (1999).

${ }^{17}$ M. L. Seaford, D. H. Tomich, K. G. Eyink, W. V. Lampert, F. E. Ejeckam, and Y.-H. Lo in 1997 IEEE International Symposium on Compound Semiconductors: Proceedings of the IEEE Twenty-fourth International Symposium on Compound Semiconductors, edited by M. Melloch and M. A. Reed. (Institute of Physics, Philadelphia, 1998), pp. 29-32.

${ }^{18}$ C. L. Chua, W. Y. Hsu, C. H. Lin, G. Christenson, and Y. H. Lo, Appl. Phys. Lett. 64, 3640 (1994).

${ }^{19}$ Handbook of Glass Properties, edited by N. P Bansal and R. H. Doremus (Academic, Orlando, FL, 1986).

${ }^{20}$ P. F. Fewster and N. Andrew, J. Phys. D: Appl. Phys. 28, A97 (1995).

${ }^{21}$ R. A. Cowley, Acta Crystallogr., Sect. A: Found. Crystallogr. 43, 825 (1987). 